\title{
Scalable, chip-based optically-controlled gates for quantum information processing
}

\author{
I. A. Burenkov \\ Physics Department, Lomonosov Moscow State University, Moscow 119991, \\ Russia

\section{O. V. Tikhonova} \\ Physics Department, Lomonosov Moscow State University, Moscow 119991, \\ Russia \\ Skobeltsyn Institute of Nuclear Physics, Lomonosov Moscow State University, \\ Moscow, Russia

\section{S. V. Polyakov} \\ National Institute of Standards and Technology, Gaithersburg, Maryland 20899, \\ USA \\ E-mail: sergey.polyakov@nist.gov
}

\begin{abstract}
Here we present a simple and robust method to build on-the-fly configurable quantum gates based on a photonic exchange between quantum nodes. The idea is based on a high reflectivity of Bragg grating structures near resonant wavelengths. The control is exerted by applying an external strongly off-resonant or even a static electromagnetic field and taking advantage of the Kerr effect. When the nonlinear phase shift is strong enough, the Bragg mirror disappears, thereby allowing a transmission of a wave packet from one node to another. An example of a protocol for quantum logic gates that relies on this
\end{abstract} framework is offered.

PACS numbers: 42.50.Ex, 42.50.Pq, 42.50.Ct

Keywords: qunatum optics, nonlinear optics, integrated optics 


\section{Introduction}

The progress of quantum information science has been very significant. On the theory side, multiple algorithms that take advantage of quantum information processing have been developed [1, 2, 3]. Multiple physical systems, particularly those based on trapped ions, neutral atoms, and quantum dots [4, 5, 6, 7] have been surveyed and found suitable as quantum nodes. To be useful, a physical carrier of quantum information should couple well with the other carriers when needed, but otherwise, must be isolated from other carriers and the environment. In practice, satisfying this condition is hard, and requires a major technical effort. At present, only a few nodes have been demonstrated to be fully controlled simultaneously [8, 9, 10]. This comes at a cost of a significant experimental overhead, impeding further growth. Yet, to support a practical quantum information algorithm, the number of gates must be large. Thus, an additional criterion arises the ability to manufacture and link a large number of nodes, and maintain a high degree of control over those nodes with little additional technical overhead to support it - or scalability. The most promising candidates for scalability are physical systems based on solid-state nodes, such as quantum dots and NV centers [5, 11. Thus, the main problem and the goal of an investigation is to combine the scalability with logic operations "on demand".

A basic quantum processing unit consists of nodes (i.e. atoms, quantum dots, etc.) that are separated from the environment (for example, with photonic crystals) and coupled to on-chip waveguides for mutual interaction and an optical readout. To date, much effort is directed towards the development of quantum information processing systems with nodes connected by passive optical interconnects. A passive optical coupling between quantum nodes simplifies the interconnection but makes the design of nodes more complex. Thus, passive optical interconnects may be more suitable for long-distance quantum communication. For a scalable quantum information transfer between nearby nodes within a quantum processing block (i.e. on a chip), it might be beneficial to use simple two-level systems as nodes and use actively-switched channels. In this letter, we propose quantum processing units that take advantage of actively controlled optical channels based on a natural property of solids - the Kerr nonlinearity. We show that actively-controlled optical channels are a simple, robust, versatile and highly scalable approach to configuring and operating quantum gates.

This manuscript is structured as follows. First, we introduce the idea of a quantum protocol based on stop-band mirrors and cross-phase modulation switching. Next, we discuss an isolated node-cavity system as an elementary block of this scheme. Thirdly, the photon exchange is discussed. Then we show how to use photon exchange and single-qubit inversion to build key two-qubit quantum gates. A feasibility study providing technical details to aid future experiments is offered as a supplementary information.

\section{Overview of the method}

Our proposed optical channel connects two or more Nlevel quantum nodes by a nonlinear Bragg waveguide, whose stop-band contains $\lambda_{1}$, the wavelength of an optical transition of the quantum nodes (see Fig. 1). Ordinarily, the high reflectivity of a Bragg grating structure (see the inset in Fig. 1) ensures isolation between the nodes, and helps maintain high-Q coupling due to Bragg reflection 12. With the grating in place, the nodes are isolated, and independent manipulation of single nodes becomes possible through deterministic interaction with a classical laser pulse. This enables single-qubit gates. We refer to this configuration as an "isolated node-cavity system". At the same time, the Bragg resonance of the nonlinear structure can be removed either with an external far off-resonant optical [13] or a static electrical field [14] (shown as a field with a wavelength $\lambda_{2}$ in Fig. 1) due to the crossphase Kerr effect. With the Bragg mirror removed, a "common node cavity", which connects two or more nodes, occurs. A common node cavity enables photon exchange between the nodes, giving rise to multi-qubit gates. Because we adopted the classical nonlinear Kerr switching for quantum gates, the range of material systems exhibiting the necessary nonlinearity is very broad. The enabling calculation and experimental results, then, are directly applicable to any of those materials, after scaling by their Kerr coefficient.

The advantage of this scheme is its high degree of versatility in real-time. That is, single-qubit or manyqubit gates are supported, they can be configured on demand and switched on and off as needed. Because a photon exchange between a pair of nodes is sufficient to support most common quantum gates, a discussion 


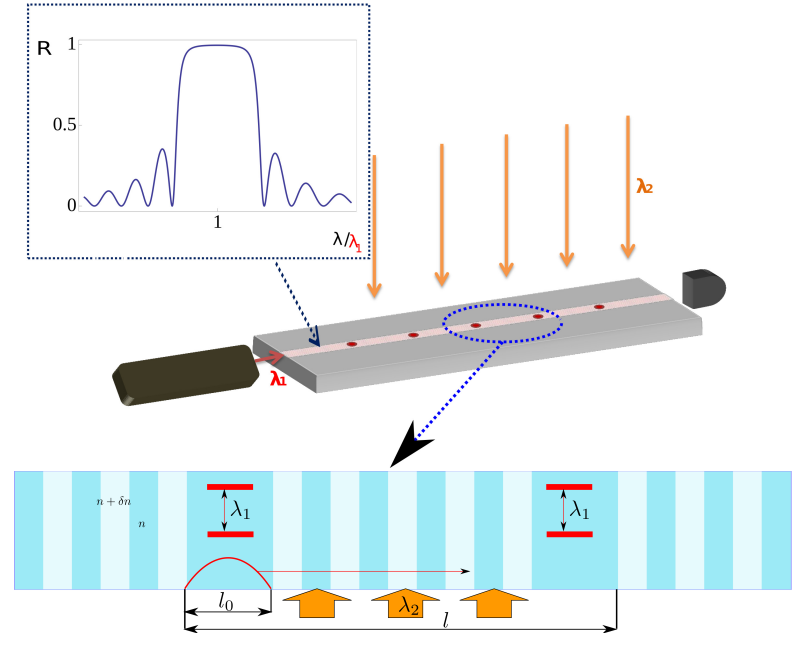

Figure 1. (Color online) A scalable 1-dimensional quantum register with a nonlinear Bragg waveguide connecting several Nlevel quantum nodes. At least one optical transition wavelength $\lambda_{1}$ between node levels $|e\rangle$ and $|g\rangle$ is in the stop band of a Bragg waveguide, turning the channel into a high-reflective cavity for efficient light-matter coupling. The Bragg structure is controlled via a cross-phase modulation due to a Kerr nonlinear response to the external laser beam $\lambda_{2}$, incident from above the chip. The inset shows the typical reflectivity dispersion curve of the Bragg structure.

of multi-node photon exchange is outside the scope of this manuscript.

Here we demonstrate our concept for quantum nodes that are single two-level quantum systems, for example, quantum dots or surface-trapped atoms. In this arrangement the system "atom+field" is used as a qubit, acting as storage, as single qubit gate and initialization/readout; whereas a pair of nodes offers two-qubit gates. We point out that protocols discussed here require strong coupling of nodes and cavities because we used the most basic, 2-level systems as nodes. The use of N-level systems would eliminate the need for strong coupling (see, for example, 15 and references therein). An adaptation of our method to N-level systems, however, is beyond the scope of this Letter.

\section{Isolated node-cavity system and one-qubit gates}

Each node is surrounded by Bragg mirrors giving rise to a set of isolated cavities with an effective length $l_{0}$. A node is coupled to one of the cavity eigenmodes: $F_{s_{0}}\left(z, l_{0}\right)=\sqrt{2 / l_{0}} \sin \left(\pi s z / l_{0}\right)$, where $s_{0}$ is the mode number. The isolated node-cavity system with one quantum of excitation undergoes vacuum Rabi oscillations between the exited state of the atom and a single excitation of the cavity mode. The coupling between the node and the cavity is given by the Rabi frequency $\Omega_{0}$, which depends on a dipole moment $d_{g e}$, a transition wavelength $\lambda_{1}$ and a mode volume $V$. The expression for Rabi frequency in SGC is:

$\Omega_{0}=d_{i f} \sqrt{\frac{8 \pi^{2} c}{\hbar V \lambda_{1}}}$,

where $\hbar$ is the Planck constant and $c$ is the speed of light in vacuum. As demonstrated by prior work on quantum dots coupled to Bragg resonators 16, 17, 18, the mode volume can be small, i.e. on the order of $(\lambda / n)^{3}$, and the dipole moment as large as $\approx 10^{-28} \mathrm{C}$. $\mathrm{m} \approx 10 \mathrm{ea}_{0}$ can be achieved, yielding the vacuum Rabi frequency of $\Omega_{0} \approx 10^{8} \div 10^{9} \mathrm{rad} \cdot \mathrm{s}^{-1}$. To achieve such a frequency [19], the transparency of Bragg mirrors in the closed channel should be less than $10^{-6}$, which is within experimental reach [20].

For a single node with vacuum Rabi oscillations, a natural choice of basis for qubit states are bipartite states of the system "atom+field": $|0\rangle=|e\rangle|0\rangle$ and $|1\rangle=|g\rangle|1\rangle$. These states can be read out in a deterministic way by creating a transparency channel between a cavity and a detector with an efficiency comparable to that of a single photon detector (For systems with more than two "atomic" levels, the qubit state can be measured differently: via a transition to an axillary exited state of the "atom"). An initialization of the nodes can be done by opening a transparency channel between a single photon source and a node, i.e. similarly to read-out. A single photon source can be made with a laser and one dedicated node, through various mechanisms described in the literature. One can use a resonant classical laser $\pi / 2$ - pulse 21 or adiabatic rapid passage 22 or a photon blockade [23]. In the first case, the resonant external laser field applied to the system provides a deterministic initialization of the node with $\pi$-pulses, $\pi / 2$-pulses, etc. With the proper choice of the intensity of the initialization classical field, the initialization time can be made several orders shorter than one period of vacuum Rabi oscillations of the node. Since initialization is an operation on a single isolated node, multiple nodes can be initialized in parallel.

Several one-qubit gates can be implemented on isolated nodes. For instance, the "inversion" operator can be implemented by freezing Rabi oscillations in selected nodes by sending a set of classical $2 \pi$ pulses 21] and allowing these nodes to remain in their initial quantum states while letting the other nodes evolve.

\section{Common node-cavities and photon exchange}

Two-qubit gates are mediated by a high-fidelity photonic exchange between the nodes. Here we describe the use of cross-phase modulation that turns 
the Bragg mirror that separates the two isolated nodes into a transparent waveguide. Once the control field is applied between a pair of nodes, the mirror disappears and the photon exchange occurs. Because a typical photon exchange duration is much faster than any interactions between the nodes and cavities, only a photonic part of a bipartite qubit will be affected. While the corresponding photon wavefunction is in an eigenmode of an isolated cavity, it is not in an eigenmode of a much larger common cavity. Then, the propagation of the photon is given by a solution of the wave equation, which has the form:

$\frac{\partial^{2} E}{\partial z^{2}}-\frac{n^{2}}{c^{2}} \frac{\partial^{2} E}{\partial t^{2}}=0$

where $n$ is the effective refractive index of the waveguide. Eq. 2 is supplemented with an initial condition $E(z, t=0)$. This condition describes a photon wave-packet immediately before the control field was applied. We have:

$E(z, t=0)=F_{s_{0}}\left(z, l_{0}\right)$,

This initial field is expanded over the set of the eigenfunctions of the common cavity with an effective length $l>l_{0}$. Within our approximations, a propagation of the initial field has an analytical solution:

$E(z, t)=\sum_{s} C_{s} F_{s}(z, l) e^{i \omega_{s} t}$

where the amplitudes $C_{s}=\left\langle F_{s}(z, l) \mid F_{s_{0}}\left(z, l_{0}\right)\right\rangle$ are the projections of the initial state on each of the eigenmodes of the common cavity and $\omega_{s}=k s c / n=$ $\pi s c /(n l), s=1,2 \ldots$

The energy distribution over different eigenmodes $F_{s}(z, l)$ of the common cavity is given by:

$W_{s}=\left|C_{s}\right|^{2}=\left[\frac{2(-1)^{s_{0}} s_{0} \sin \left(\frac{\pi l_{0} s}{l}\right)}{\left(\frac{1}{l}\right)^{3 / 2} \sqrt{\frac{1}{l_{0}}}\left(\pi l^{2} s_{0}^{2}-\pi l_{0}^{2} s^{2}\right)}\right]^{2}$.

Note that this distribution strongly depends on the ratio $l / l_{0}$ and the mode number of the populated mode in the isolated cavity $s_{0}$. Some examples of normalized to have equal integral populations $W_{s}$ are shown in Fig. 2 for different $s_{0}=1, \ldots, 10$ and for $l / l_{0}=200$. The distribution becomes narrower for larger values of $s_{0}$. A narrower distribution may be beneficial in material systems where it is difficult to avoid dispersion. On the other hand, coupling to higher modes decreases the coupling strength (i.e. the Rabi frequency in (1)). Note the tradeoff between the coupling constant which decreases as $\sqrt{s_{0}}$, and the width of the distribution over eigenmodes, which narrows linearly with $s_{0}$.

We now include dispersion by introducing $\omega_{s}=$ $(\pi c / n)\left((s / l)+\left(c D / n^{2}\right)\left(1-s / s_{r}\right)^{2}\right)$ into eq. (4), where $D$ is a group-velocity dispersion parameter and $s_{r}=$ $s_{0} l / l_{0}$ is the mode number of the resonant mode of the

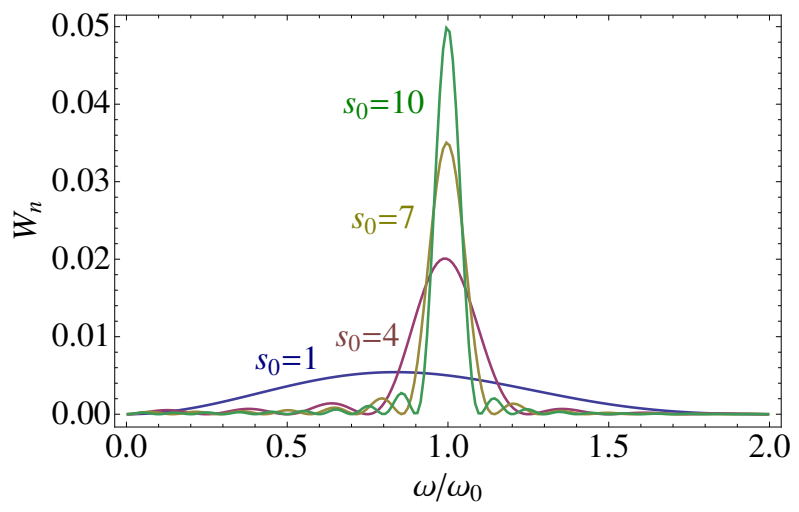

Figure 2. Normalized population of different eigenmodes $F_{s}(z, l)$ of the common resonator for different initially populated modes $s_{0}$ of the node cavity

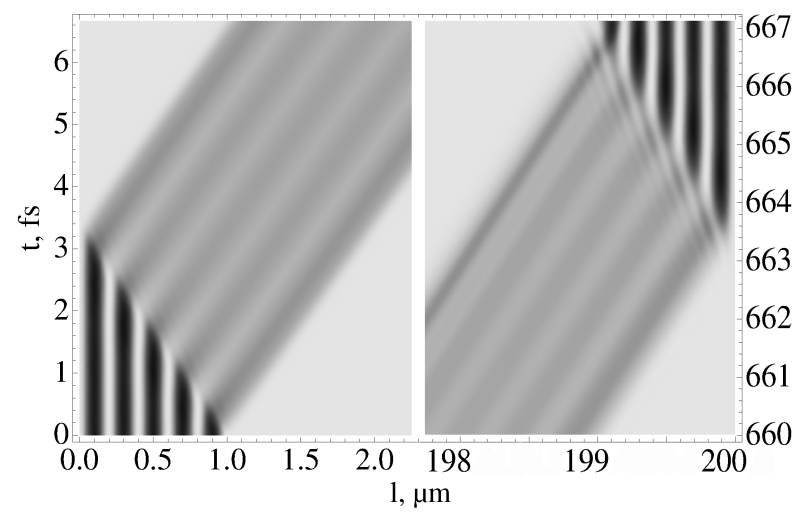

Figure 3. Nearly-faithful restoration of field distribution $E(z, t=0)$ for an isolated cavity's mode $s_{0}=10$ after a propagation between the nodes in a dispersive waveguide (dispersion parameter $D=10 \mathrm{ps} /(\mathrm{nm} \cdot \mathrm{km})$ ).

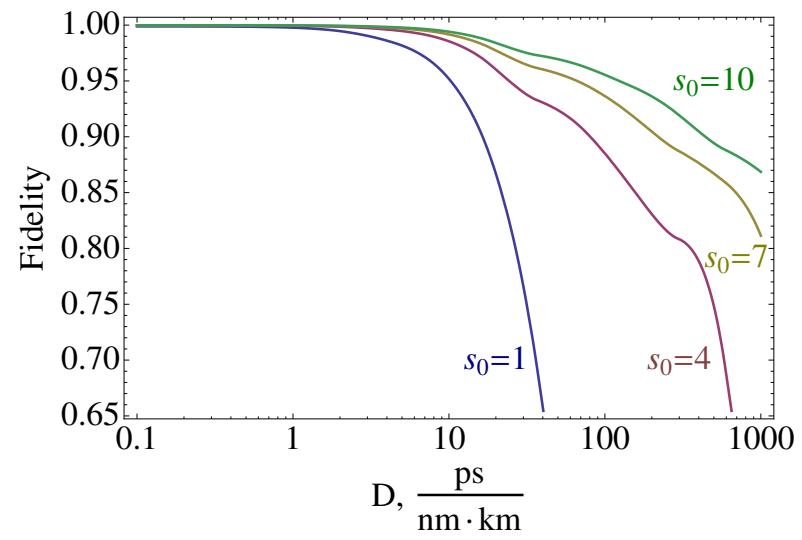

Figure 4. Fidelity for a round-trip photon wave-packet transfer (from one node to another and back) as a function of the group-velocity dispersion parameter $D$ for different isolated cavity modes $s_{0}$.

common cavity. Because we consider eigenmodes of a node resonator, waveguide dispersion does not change 
the standing wave field shape, i.e. when the field $\lambda_{2}$ is off. Figure 3 illustrates how the wave packet with $\lambda_{1}$ propagates during the photon exchange between the two nodes, where the stop band in-between the nodes is removed via a classical control optical field $\lambda_{2}$ with dispersion $D=10 \mathrm{ps} /(\mathrm{nm} \cdot \mathrm{km})$. It is evident from Figure 3 that at the end of the transfer, the wavepacket has traveled to the target node with nearly unchanged amplitude and phase profiles. Therefore, upon propagation, a photon can readily interact with an "atom" in the target node once the control field is switched off.

Our subsequent simulation illustrates the effect of dispersion on fidelity $F$ of node-to-node photon state transfer. Because we use normalized eigenfunctions as initial conditions for (2), $F=$ $\operatorname{Re}\left[\int_{0}^{l_{0}} E(z, t=2 l /(c / n))^{*} E(z, t=0) d x\right]$. We see that the higher the mode of an isolated cavity $s_{0}$ is used; the higher dispersion coefficients can be tolerated, Fig. 4. In particular, for a node-to-node transfer described above and $s_{0}=1$ the calculated fidelity $F>0.99$ for $D<2 \mathrm{ps} /(\mathrm{nm} \cdot \mathrm{km})$, while for $s_{0}=10$ dispersion coefficients up to $D=20 \mathrm{ps} /(\mathrm{nm} \cdot \mathrm{km})$ yield $F \approx 0.99$, a ten-fold reduction in dispersion spreading. Fidelity monotonically decreases as the group velocity dispersion increases. Ripples are seen in Fig. 4 due to the interference effects.

\section{Two-qubit gate protocols}

Using isolated node manipulations and the controlled photon exchange between nodes we have developed simple and scalable protocols for basic quantum gates: SWAP, CNOT, and phase rotation. We define the two basic operations supported by the framework of an actively-switched photonic network and required to build quantum gates. We assume that the two cubits are stored in the two adjacent isolated cavities, labeled 1 and 2. First, a photon exchange between cavities through a common cavity is denoted by an exchange operator:

$\hat{\leftrightarrow}\left|a_{1}\right\rangle_{1}\left|n_{1}\right\rangle_{1}\left|a_{2}\right\rangle_{2}\left|n_{2}\right\rangle_{2}=\left|a_{1}\right\rangle_{1}\left|n_{2}\right\rangle_{1}\left|a_{2}\right\rangle_{2}\left|n_{1}\right\rangle_{2}$

where $|a\rangle_{i}$ is an atomic state and $|n\rangle_{i}$ is a photon number state in the $i^{\text {th }}$ cavity. Note that this procedure only exchanges the photonic part of the two bipartite cubits, leaving the node excitation part intact. Second, an inversion operator is naturally provided by Rabi oscillations in the nodes. When $2 \pi$-pulses are applied to the selected group of nodes to freeze their evolution, the other nodes undergo an inversion:

$\hat{\pi}|g\rangle|n\rangle=|e\rangle|n-1\rangle$
$\hat{\pi}|e\rangle|n\rangle=|g\rangle|n+1\rangle$
Table 1. Protocols for SWAP and CNOT operations

\begin{tabular}{|c|c|c|c|}
\hline \multicolumn{3}{|c|}{ SWAP } \\
\hline$\hat{O p} . \mid$ & $|g\rangle_{1}|1\rangle_{1}|g\rangle_{2}|1\rangle_{2}|g\rangle_{1}|1\rangle_{1}|e\rangle_{2}|0\rangle_{2}|e\rangle_{1}|0\rangle_{1}|g\rangle_{2}|1\rangle_{2}|e\rangle_{1}|0\rangle_{1}|e\rangle_{2}|0\rangle_{2}$ \\
$\hat{\leftrightarrow}$ & $|g\rangle_{1}|1\rangle_{1}|g\rangle_{2}|1\rangle_{2}|g\rangle_{1}|0\rangle_{1}|e\rangle_{2}|1\rangle_{2}|e\rangle_{1}|1\rangle_{1}|g\rangle_{2}|0\rangle_{2}|e\rangle_{1}|0\rangle_{1}|e\rangle_{2}|0\rangle_{2}$ \\
$\hat{\pi}$ & $|e\rangle_{1}|0\rangle_{1}|e\rangle_{2}|0\rangle_{2}|g\rangle_{1}|0\rangle_{1}|g\rangle_{2}|2\rangle_{2}|g\rangle_{1}|2\rangle_{1}|g\rangle_{2}|0\rangle_{2}|g\rangle_{1}|1\rangle_{1}|g\rangle_{2}|1\rangle_{2}$ \\
$\hat{\leftrightarrow}$ & $|e\rangle_{1}|0\rangle_{1}|e\rangle_{2}|0\rangle_{2}|g\rangle_{1}|2\rangle_{1}|g\rangle_{2}|0\rangle_{2}|g\rangle_{1}|0\rangle_{1}|g\rangle_{2}|2\rangle_{2}|g\rangle_{1}|1\rangle_{1}|g\rangle_{2}|1\rangle_{2}$ \\
$\hat{\pi}$ & $|g\rangle_{1}|1\rangle_{1}|g\rangle_{2}|1\rangle_{2}|e\rangle_{1}|1\rangle_{1}|g\rangle_{2}|0\rangle_{2}|g\rangle_{1}|0\rangle_{1}|e\rangle_{2}|1\rangle_{2}|e\rangle_{1}|0\rangle_{1}|e\rangle_{2}|0\rangle_{2}$ \\
$\hat{\leftrightarrow}$ & $|g\rangle_{1}|1\rangle_{1}|g\rangle_{2}|1\rangle_{2}|e\rangle_{1}|0\rangle_{1}|g\rangle_{2}|1\rangle_{2}|g\rangle_{1}|1\rangle_{1}|e\rangle_{2}|0\rangle_{2}|e\rangle_{1}|0\rangle_{1}|e\rangle_{2}|0\rangle_{2}$ \\
\hline \hline \multicolumn{3}{|c|}{ CNOT } \\
\hline$\hat{O} p . \mid$
\end{tabular}

where $\hat{\pi}$ - is a delay equal to one half of Rabi oscillations period during the evolution of a node. In the Table, $\hat{\pi}$ refers to an inversion on both the nodes, $\hat{\pi}_{i}$, where $i=1,2$ denotes an inversion of just the first (second) node, while the other one is not inverted.

The above operators are sufficient to implement SWAP and CNOT gates. The associated protocols are shown in Table 1

\section{Conclusions}

We have introduced a method of implementing scalable light-controlled gates for quantum information processing, based on N-level systems (nodes) exchanging photons via optically-controlled nonlinear Bragg waveguides. This method for all-optical switching relies on cross-phase modulation that removes the effective Bragg resonance and creates transparency so that the control field can be detuned very far from any of the node's resonances. Because the control field $\lambda_{2}$ is a classical field, multiple pairs of nodes may be controlled simultaneously. Thus, quantum information processing can be made massively parallel. This design can be implemented on a chip with the currently existing technology. A particular experimental implementation of this method is described in the Supplementary material section. Note, that our proposal only requires a fast (femtosecond scale) classical switch. The search for fast, high-contrast optically controlled switches is a very active field of research, 24, 25, 26, 27, 28, There- 
fore one should expect alternative experimental realizations of the proposed protocol.

While, in our manuscript, we assumed that the control field is applied perpendicular to the waveguide, other configurations are also possible. To implement even more exotic, multi-body based algorithms [29, nodes can be arranged in one-, two-, or threedimensional structures. This opens the way to implement topologically protected and/or massively parallel quantum interactions, commonly studied with cold atoms in optical lattices, on a solid state chip.

\section{SUPPLEMENTARY MATERIAL}

We discussed the general idea and introduced protocols for a quantum circuit based on actively switched optical channels. Because a broad range of materials and geometries can be employed to implement these circuits [30, 31, 32, 33] we presented general design features for such channels. Here we offer a detailed study of one possible implementation of our protocol, based on a lithium niobate waveguide. The purpose of this study is twofold. First, it provides guidance for an enabling experiment with Bragg switches. Second, it introduces certain technological enhancements that are aimed at scalability.

Lithium niobate is commonly used in optical devices and is relatively well understood. We consider it a good candidate for an experimental realization because it has one of the largest Kerr nonlinearity constants $n_{2}=83.310^{-16} \mathrm{~cm}^{2} / \mathrm{W}$ 34. All necessary fabrication techniques required for realization of our proposal, particularly the making of the waveguides [20], the permanent modulation of the refraction index 35, 36, the making of the dynamic gratings with a static electrical field 14 and placing the conductive nanostructures 37 were demonstrated. The making of the dynamic gratings with a light field [13] as well as field enhancement with periodic conductive nanostructures 38. were demonstrated in other materials, but are compatible with lithium niobate.

The overall geometry of the structure and the mode profile is shown in Fig. 1 of the manuscript. The waveguide can be implemented in lithium niobate with an average diameter of $\approx 500 \mathrm{~nm}$ on a lower refractive index (RI) substrate with. Periodic variation of the RI of the waveguide can be implemented statically in several different ways or dynamically induced by a control field. An RI contrast is chosen based on the nonlinear constant, available control field power and the required bandgap width.

To implement the proposed switching mechanism in a lithium niobate waveguide, a low RI contrast should be used, such that a control field of $\approx$
$10^{10} \ldots 10^{11} \mathrm{~W} / \mathrm{cm}^{2}$ can erase a static Bragg grating (BG). This intensity corresponds to a refractive index change due to a Kerr cross-phase modulation of $\approx$ $10^{-3}$. To make a high $\mathrm{Q}$ cavity with such a low RI contrast, the length of the grating structure should be quite long: $\approx 3000$ periods. Notice that the bandgap width will also decrease $(\delta \lambda \approx 0.5 \mathrm{~nm}$, thus a higher longitudinal cavity mode should be coupled to the quantum node to provide adequately high reflectivity $\left(s_{0} \approx 100\right)$. To erase a BG structure, the control field should have a spatial pattern that corresponds to the periodic BG structure. An array of plasmonic antennas along the waveguide could be used to enhance the local optical field and provide modulation [39, 38, thus reducing the power required and simplifying the preparation of the control beams. The effect of placing the antennas is shown in Fig. 5, where a numerical calculation was performed with an FDTD method for a control field with a wavelength $\lambda=1 \mu \mathrm{m}$. As a result, a simple flat-top (and unmodulated) control field can be applied as a control, further aiding scalability. In addition, such an array significantly decreases the power requirements for the control field. Eliminating the BG altogether is advantageous because it reduces undesired dispersion effects. We have modeled the propagation of a wave packet through the waveguide and it is evident that dispersion in this system does not significantly distort the wavepacket, yielding a fidelity above 0.99 for the $10^{t h}$ mode (c.f. Fig. 4 of the manuscript). As seen in that figure, the fidelity improves further at even higher mode numbers. Placing an array of conducting particles in the vicinity of the waveguide does not significantly increase the propagation loss because the $\lambda_{1}$ field is far off-resonant for gold nanoantennas.

Assuming that the control field is sufficiently detuned from the transition frequency used in the 2level nodes $(\Delta \lambda>100 \mathrm{~nm})$, a qubit dipole moment of $\approx e a_{0}$ (where $a_{0}$ is Bohr radius) and a resonant energy of $\approx 1 \mathrm{eV}$ [40, the resulting Rabi oscillations of the qubit due to the control field yield an amplitude that is less than $10^{-3}$, i.e. negligible on the time scale in question. Minor drawbacks of using shallow index contrast vs. high index contrast are the relatively long distances between nodes, longer times for gate operations, and longer Rabi periods for the coupling between nodes and isolated cavities (up to 10 times in comparison to the lowest longitudinal mode attainable with high index contrast).

On the other hand, high RI contrast, for instance $\delta n=0.04$, would result in a wide band-gap $(\delta \lambda \approx 10$ $\mathrm{nm}$, providing finesse $f \approx 10^{6}$ for $10^{\text {th }}$ field mode) with only 200 periods. When a large index contrast is employed, an optically controlled bandgap shift, rather than a BG erasure, should be used to lower 


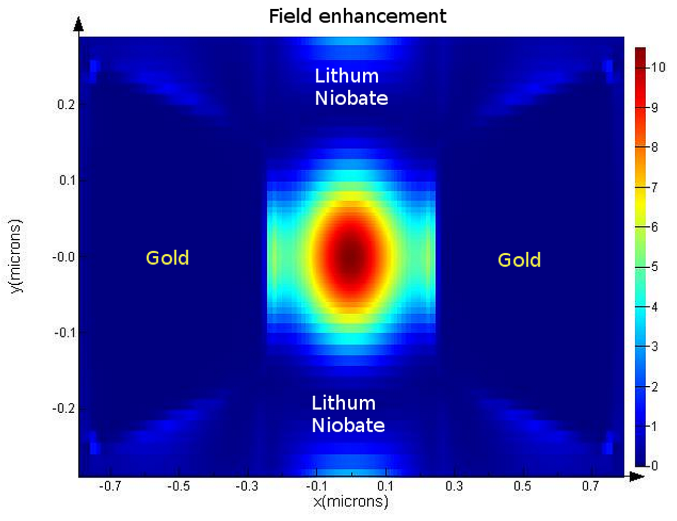

Figure 5. Field intensity enhancement in $\mathrm{LiNbO}_{3}$ waveguide due to plasmonic resonance of gold nanoantenna (dark blue trapezoidal regions) with air cladding

switching power requirements. Remarkably, a required control field pulse peak power of $\approx 720 \mathrm{~W}$ and a duration of $\approx 100 \mathrm{ps}$ is sufficient to achieve bandgap shifts of $\approx 0.6 \mathrm{~nm}$ in barium fluoride [41, 26]. Due to a larger Kerr coefficient, this power is reduced more than 30 -fold in lithium niobate. To reduce the role of strong dispersion as the light propagates in the medium with an optically detuned bandgap, a higher-order longitudinal isolated cavity mode should be employed (see Fig. 2 of the manuscript), and/or dispersion compensation techniques should be applied. Dispersion engineering can be achieved e.g. through advanced 2D patterning of lithium niobate [42.

In conclusion, we discussed general design rules using one possible experimental implementation of the platform as an example. In addition an array of plasmonic antennas along the waveguide enhances the local optical field and provides modulation, thus aids scalability.

\section{ACKNOWLEDGEMENTS}

Authors thank Mohammad Hafezi for fruitful discussions and critical comments. SVP thanks Garnett Bryant for encouraging remarks. IAB and OVT acknowledge a partial support by Russian Foundation for Basic Research grant No 14-02-00389_a.

\section{References}

[1] Ekert A and Jozsa R 1998 Philosophical Transactions of the Royal Society of London A: Mathematical, Physical and Engineering Sciences 356 1769-1782 ISSN 1364-503X

[2] Shor P 2004 Quantum Information Processing 3 513 ISSN 1570-0755 URL http://dx.doi.org/10.1007/ s11128-004-3878-2

[3] DiVincenzo D P 1995 Science 270 255-261

[4] Home J P, Hanneke D, Jost J D, Amini J M, Leibfried D and Wineland D J 2009 Science 325 1227-1230 ISSN 0036-8075

[5] Gazzano O and Solomon G S 2016 J. Opt. Soc. Am. B 33 C160-C175 URL http://josab.osa.org/abstract.cfm? URI=josab-33-7-C160

[6] Saffman M, Walker T G and Mölmer K 2010 Rev. Mod. Phys. 82 2313-2363 URL http://link.aps.org/doi/ 10.1103/RevModPhys.82.2313

[7] Monroe C 2002 Nature 416 238-246 ISSN 0028-0836 URL http://dx.doi.org/10.1038/416238a

[8] Kielpinski D, Monroe C and Wineland D J 2002 Nature 417 709-711 ISSN 0028-0836

[9] Mavadia S, Goodwin J F, Stutter G, Bharadia S, Crick D R, Segal D M and Thompson R C 2013 Nat Commun 4 URL http://dx.doi.org/10.1038/ncomms357110.1038/ ncomms 3571

[10] Sahling S, Remenyi G, Paulsen C, Monceau P, Saligrama V, Marin C, Revcolevschi A, Regnault L P, Raymond S and Lorenzo J E 2015 Nat Phys 11 255-260 ISSN 1745-2473 article URL http://dx.doi.org/10.1038/nphys3186

[11] Dutt M V G, Childress L, Jiang L, Togan E, Maze J, Jelezko F, Zibrov A S, Hemmer P R and Lukin M D 2007 Science 316 1312-1316 (Preprint http://www.sciencemag.org/ content/316/5829/1312.full.pdf) URL http://www. sciencemag.org/content/316/5829/1312. abstract

[12] Nozaki K, Tanabe T, Shinya A, Matsuo S, Sato T, Taniyama H and Notomi M 2010 Nature Photonics 4 477-483 ISSN 1749-4885 URL http://www.nature.com/ doifinder/10.1038/nphoton.2010.89

[13] Schneider T, Wolfframm D, Mitzner R and Reif J 1999 Applied Physics B 68 749-751 ISSN 0946-2171 URL http://dx.doi.org/10.1007/s003400050698

[14] Jin W, Chiang K S and Liu Q 2008 Opt. Express 1620409 20417 URL http://www.opticsexpress.org/abstract. cfm?URI=oe-16-25-20409

[15] Luo L, Hayes D, Manning T, Matsukevich D, Maunz P, Olmschenk S, Sterk J and Monroe C 2009 Fortschritte der Physik 57 1133-1152 ISSN 1521-3978 URL http: //dx.doi.org/10.1002/prop.200900093

[16] Pelton M, Santori C, Vuckovic J, Zhang B Y, Solomon G S, Plant J and Yamamoto Y 2002 Phys. Rev. Lett. 89233602 ISSN 0031-9007

[17] Reithmaier J P, Sek G, Loffler, Hofmann C, Kuhn S, Reitzenstein S, Keldysh L V, Kulakovskii V D, Reinecke T L and Forchel A 2004 Nature 432 197-200 URL http://www.nature.com/nature/journal/v432/n7014/ abs/nature02969.html

[18] Yoshie T, Scherer A, Hendrickson J, Khitrova G, Gibbs H M, Rupper G, Ell C, Shchekin O B and Deppe D G 2004 Nature 432 200-203 URL http: //www.nature.com/ nature/journal/v432/n7014/abs/nature02969.html

[19] Bose R, Cai T, Choudhury K R, Solomon G S and Waks E 2014 Nature Photonics 8 858-864 ISSN 1749-4885 URL http://www . nature . com/doifinder/10. 1038/nphoton.2014.224

[20] Pierno L, Dispenza M, Secchi A, Fiorello A and Foglietti V 2008 Journal of Optics A: Pure and Applied Optics 10 064017 URL http://stacks.iop.org/1464-4258/10/i= $6 / a=064017$

[21] Allen L and Eberly J H 1987 Optical Resonance and TwoLevel Atoms (Dover: New York)

[22] Wei Y J, He Y M, Chen M C, Hu Y N, He Y, Wu D, Schneider C, Kamp M, Hfling S, Lu C Y and Pan J W 2014 Nano Letters 14 65156519 pMID: 25357153 (Preprint http://dx.doi.org/ 10.1021/nl503081n) URL http://dx.doi.org/10.1021/ n1503081n

[23] Birnbaum K M, Boca A, Miller R, Boozer A D, Northup T E and Kimble H J 2005 Nature 436 87-90 ISSN 00280836 URL http://dx.doi .org/10.1038/nature03804 
[24] Midwinter J, Liao P and Kelley P 2012 Photonics in Switching Quantum Electronics-Principles and Applications (Elsevier Science) ISBN 9780080924748 URL https://books.google.com/books?id=WYBY JpET-dwC

[25] Sivan Y, Ctistis G, Yüce E and Mosk A P 2015 Opt. Express 23 16416-16428 URL http://www.opticsexpress.org/ abstract.cfm?URI=oe-23-12-16416

[26] Zang Z and Zhang Y 2012 Appl. Opt. 51 3424-3430 URL http://ao.osa.org/abstract.cfm?URI=ao-51-16-3424

[27] Tada K 2005 Photonics Based on Wavelength Integration and Manipulation IPAP books (IPAP) ISBN 9784900526198 URL https://books.google.com/ books?id=tTlzAQAACAAJ

[28] Eichler H, Günter P and Pohl D 1986 Laser-induced Dynamic Gratings Exploration of the Deep Continental Crust (Springer-Verlag) ISBN 9780387158754 URL https://books . google.com/books?id=N3lrQgAACAAJ

[29] Greiner M, Mandel O, Esslinger T, Hansch T W and Bloch I 2002 Nature 415 39-44 ISSN 0028-0836 URL http://dx.doi.org/10.1038/415039a

[30] Vukovic N, Healy N, Suhailin F H, Mehta P, Day T D, Badding J V and Peacock A C 2013 Sci Rep 32885 ISSN 2045-2322 24097126[pmid] URL http://www.ncbi. nlm.nih.gov/pmc/articles/PMC3791441/

[31] Yoshiki W and Tanabe T 2014 Opt. Express 22 2433224341 URL http://www.opticsexpress.org/abstract. cfm?URI=oe-22-20-24332

[32] Thyrrestrup H, Yce E, Ctistis G, Claudon J, Vos W L and Grard J M 2014 Applied Physics Letters 105111115

[33] De Angelis C, Modotto D, Locatelli A and Wabnitz S 2015 Optical Guided Wave Switching (Cham: Springer International Publishing) pp 71-104 ISBN 978-3-31914992-9

[34] Nikogosian D N 1997 Properties of Optical and LaserRelated Materials: A Handbook (Wiley Interscience)

[35] Paipulas D, Malinauskas M, Smilgeviius V and Sirutkaitis V 2011 Permanent volume bragg grating fabrication in pure lithium niobate crystal using direct laser writing technique Lasers and Electro-Optics Europe (CLEO EUROPE/EQEC), 2011 Conference on and 12th European Quantum Electronics Conference pp 11 ISSN Pending

[36] Horn W, Kroesen S, Herrmann J, Imbrock J and Denz C 2012 Opt. Express 20 26922-26928 URL http://www.opticsexpress.org/abstract.cfm?URI= oe-20-24-26922

[37] Green T A 2007 Gold Bulletin 40 105-114 ISSN 2190-7579 URL http://dx.doi.org/10.1007/BF03215566

[38] Février M, Gogol P, Barbillon G, Aassime A, Mégy R, Bartenlian B, Lourtioz J M and Dagens B 2012 Opt. Express 20 17402-17409 URL http://www .opticsexpress. org/abstract.cfm?URI=oe-20-16-17402

[39] Bruck R and Muskens O L 2013 Opt. Express 2127652 27661 URL http://www.opticsexpress.org/abstract. cfm?URI=oe-21-23-27652

[40] Delone N and Kraĭnov V 2000 Multiphoton Processes in Atoms: Second Edition Atoms and plasmas (Springer) ISBN 9783540646150 URL https://books .google.com/ books?id=EJhSVHGe5DMC

[41] Yokota H, Kobayashi M, Mineo H, Kagawa N, Kanbe H and Sasaki Y 2008 Optics Communications 2814893 4898 ISSN 0030-4018 URL http://www. sciencedirect. com/science/article/pii/S003040180800597X

[42] Broderick N G R, Ross G W, Offerhaus H L, Richardson D J and Hanna D C 2000 Phys. Rev. Lett. 84(19) 4345-4348 URL http://link.aps.org/doi/10.1103/PhysRevLett. 84.4345 